\title{
Stemmführung nach Brunkow
}

Die Stemmführung nach Brunkow, jetzt Akrodynamik, ist ein ganzheitliches Bahnungssystem, basierend auf der Grundlage idealmotorischer Bewegungsmuster und wurde von der Krankengymnastin Roswitha Brunkow (gest. 1975) erarbeitet. Sie entwickelte eine überwiegend statische Muskelanspannungstechnik als Bahnungssystem, mit der unzureichende Muskelfunktion und Haltungsschwäche oder Haltungsfehler behandelt werden können. Über vorgegebene Winkelstellungen der Gelenke und unter Augenkontrolle des Patienten wird durch gleichzeitiges willkürliches Anspannen von Beuge- und Streckmuskeln (Kokontraktion) gegen einen imaginären Widerstand ,gestemmt“. Durch das „Einstemmen“ erfolgt eine Weiterleitung der Muskelspannung in den Rumpf, die ihrerseits eine unwillkürliche Aufrichtung des Rumpfes mit einer Ganzkörperspannung auslöst. Durch manuelle Reize des Therapeuten wie Druck-Stauch-Impulse und Wisch- und Streichtechniken kann die Muskelspannung optimiert und die gewünschte Muskelkette angeregt werden. Bewegt der Patient über die aufgebaute Spannung hinaus die eingestemmten Arme oder Beine, so lässt sich diese Spannung steigern. So soll sich eine Haltungsstörung des Rumpfes oder der Wirbelsäule in optimaler Weise durch massiven symmetrischen Einstrom propriozeptiver Impulse aus den Armen oder Beinen ausgleichen lassen (Jung et al. 1976; Paschen K 2006). Ein Vergleich mit der Vojta Therapie drängt sich auf, da auch hier die Therapie basiert auf Reflexmotorik. 